Check for updates

Cite this: RSC Adv., 2017, 7, 40095

\title{
Evaluation of structure-activity relationships of ginsenosides against amyloid $\beta$ induced pathological behaviours in transgenic Caenorhabditis elegans $\dagger$
}

\author{
Mu Zhang, (D) Fei Qian,\$ Qingling Liu, Cheng Qian, Pyone Myat Thu, Yanyan Wang, \\ Zu-Guo Zheng, Hua Yang, Ping Li (iD * and Xiaojun $\mathrm{Xu}^{*}$
}

Amyloid $\beta(A \beta)$ induced toxicity has been postulated to initiate synaptic loss and subsequent neuronal degeneration in Alzheimer's disease (AD). The activities of ginsenosides against AD are widely reported, however, a systematic in vivo study comparing the effects of different ginsenosides on $A \beta$ induced toxicity and cognitive impairment has not been described. In addition, the correlation between molecular structures and their anti-A $\beta$ activities remains uncovered. In the present study, the in vivo anti$A \beta$ effects of 17 ginsenosides were explored using three transgenic Caenorhabditis elegans (C. elegans) models that exhibits pathological behaviors associated with $A \beta$. We found that (1) ginsenoside Rc, Rd, 20(S)-Rg3, Compound $\mathrm{K}(\mathrm{CK})$ and Rg1 could significantly reduce $A \beta$ deposits, (2) ginsenoside Rc, Rd, CK, Rk3 and Rg1 inhibit $A \beta$ induced paralysis, and (3) ginsenosides 20(S)-Rg3, CK and Rk3 prevented $A \beta$ induced defects in associative learning capacity and showed antioxidative activity both in vitro and in vivo. The structure-activity relationships of 17 ginsenosides were investigated by comparing their anti-A $\beta$ effects in $C$. elegans models. Results showed that the protopanaxadiol-type has higher activities than the protopanaxatriol-type or oleanane-type; the anti-A $\beta$ effects of ginsenosides are inversely related to the sugar numbers. The results of the present study gain insight into the in vivo protective effects of ginsenosides against $A \beta$ induced toxicity, and provide useful information for the clinical use of ginsenosides.

Received 21st May 2017

Accepted 18th July 2017

DOI: $10.1039 / c 7 r a 05717 b$

rsc.li/rsc-advances
The protopanaxadiol (PPD)-type saponins with sugar moieties at the C-3 and/or C-20, the protopanaxatriol (PPT)-type saponins with sugar moieties attached to the C-6 and/or at C-20, and the oleanane-type with a nonsteroidal structure. ${ }^{11}$ Some rare ginsenosides are generated from naturally occurring types through heating, ${ }^{6,12}$ enzymatic, ${ }^{13}$ microbial $^{14}$ or chemical treatment. ${ }^{12}$ Several ginsenosides, including $\mathrm{Rb} 1,{ }^{15-17} \mathrm{Rg} 1,{ }^{18} \mathrm{Rg} 2,{ }^{19} \mathrm{Rg} 3{ }^{, 0}$ $\mathrm{Rd},{ }^{21-24} \mathrm{Re},{ }^{25} \mathrm{Rh} 1,{ }^{26} \mathrm{Rh} 2$ (ref. 27 ) and $\mathrm{CK},{ }^{28}$ have been reported to have potential benefits to improve cognition through (1) regulating the synaptic plasticity, ${ }^{16-18,28}$ (2) inhibiting neuroinflammation,,$^{20,25}(3)$ enhancing the production of neurotrophic factors,$^{24}(4)$ regulating the homeostasis of $A \beta,,^{23,27}$ (5) promoting the release of acetylcholine from hippocampus ${ }^{15}$ and other mechanisms. While to our best knowledge, there is no systematic in vivo study comparing the effects of different ginsenosides on $\mathrm{A} \beta$ induced toxicity and cognitive impairment. In addition, the correlation between structures and anti-A $\beta$ activities still has not been fully elucidated.

Since $\mathrm{AD}$ is a complex neurodegenerative disease, the cultured mammalian cell models that lack the organism complexity involved in $\mathrm{AD}$ are not suitable to fully evaluate the anti-AD activity of candidate compounds. On the other hand, the use of a transgenic mice model of $\mathrm{AD}$ for pharmacological
State Key Laboratory of Natural Medicines, China Pharmaceutical University, No. 24 Tongjia Lane, Nanjing 210009, Jiangsu, China. E-mail: xiaojunxu@cpu.edu.cn; liping@cpu.edu.cn; Fax: +86 25 83271379; Tel: +86 2583271379

$\dagger$ Electronic supplementary information (ESI) available. See DOI: 10.1039/c7ra05717b

\$ These authors contribute equally to this work. 
evaluation and mechanistic studies is time and labor consuming. Simple invertebrate models of neurodegenerative diseases offer experimental advantages for addressing basic cellular processes that are conserved among all animals. ${ }^{29}$ In the present study, by using several transgenic AD Caenorhabditis elegans (C. elegans) models, ${ }^{30}$ we explored the in vivo anti-A $\beta$ effects of 17 ginsenosides (Fig. 5, Tables 2-4), and discussed the possible structure-activity relationship based on types of aglycones and sugar moieties.

\section{Materials and method}

\subsection{Reagents}

Reference ginsenosides Rb1, Rb2, Rb3, Rc, Rd, Re, 20(S)-Rh1, Rg1, Rg2, 20(S)-Rg3, Rg6, Rk3, Ro, CK, PPD, PPT and oleanolic acid (OA) were purchased from Must Biological Technology Co. Ltd. (Chengdu, China). Diacetyl and donepezil hydrochloride (Dop) were purchased from Tokyo Chemical Industry (Tokyo, Japan). Thioflavine T (ThT), sodium azide, vitamin E (VitE) and ethanol were purchased from Sigma-Aldrich (St. Louis, MO, USA). Stock solution of all candidate drugs were made with DMSO (Sigma-Aldrich, St. Louis, MO, USA), and diluted by M9 buffer to final concentrations in C. elegans experiments, or diluted by cell culture medium in cell treatment. The final concentration of DMSO did not exceed $0.1 \%$. Diacetyl was prepared immediately before to any experiments by dilution in ethanol. Sodium azide was distilled in water to obtain a final concentration of $1 \mathrm{M} .^{31}$

\subsection{C. elegans strains}

C. elegans strains used in this study are listed in Table 1. CL4176 (smg-1ts [myo-3/A $\beta_{1-42}$ long 3'-untranslated region (UTR)]), CL802 (smg-1(cc546) I; rol-6(su1006) II), CL2006 (dvIs2[pCL12(unc-54/ human $\mathrm{A} \beta_{1-42}$ minigene) + pRF4]), CL2122 (dvIs15[(pPD30.38) unc-54(vector) + (pCL26) mtl-2:GFP]) were obtained from Caenorhabditis Genetics Center (University of Minnesota, Minneapolis, MN). CL2241 (smg-1(cc546ts); $\quad d v l s 29\left(\mathrm{pCL} 45\left(s n b-1 / \mathrm{SP}: \mathrm{A} \beta_{1-42}\right.\right.$ minigene/long $3^{\prime}$ UTR) + pRF4 (rol-6(su1006)), ${ }^{30}$ CL2109 (dvIs [pDF1 (unc-54/SP:A $\beta_{1-42}$ dimer minigene + pRF4 (rol-6(su1006))) are kind gifts from Professor Christopher D. Link (Institute for Behavioral Genetics, University of Colorado).

\subsection{C. elegans maintenance and treatment}

Nematodes were cultured in temperature controlled incubator. All strains were maintained at $16{ }^{\circ} \mathrm{C}$, and propagated on solid

Table 1 Description of the characterized transgenic $C$. elegans used in the study

\begin{tabular}{llll}
\hline Strains & Transgene & Expression & Phenotype \\
\hline CL802 & $m y o-3$ & Control for CL4176 & Wt. movement \\
CL4176 & $m y o-3 / \mathrm{A} \beta_{1-42}$ & Inducible muscle & Rapid paralysis \\
CL2109 & unc-54/A $\beta$ dimer & Control for CL2006 & No A $\beta$ formation \\
CL2006 & unc-54/A $\beta_{1-42}$ & Constitutive muscle & Progressive paralysis \\
CL2122 & $m t l-2 / \mathrm{GFP}$ & Control for CL2241 & Wt. movement \\
CL2241 & snb-1/A $\beta_{1-42}$ & Inducible neuronal & Wt. movement
\end{tabular}

A

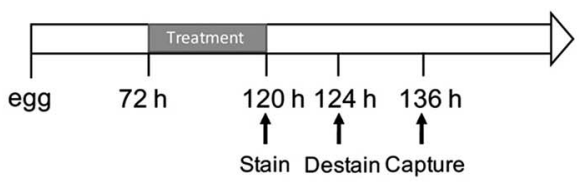

B
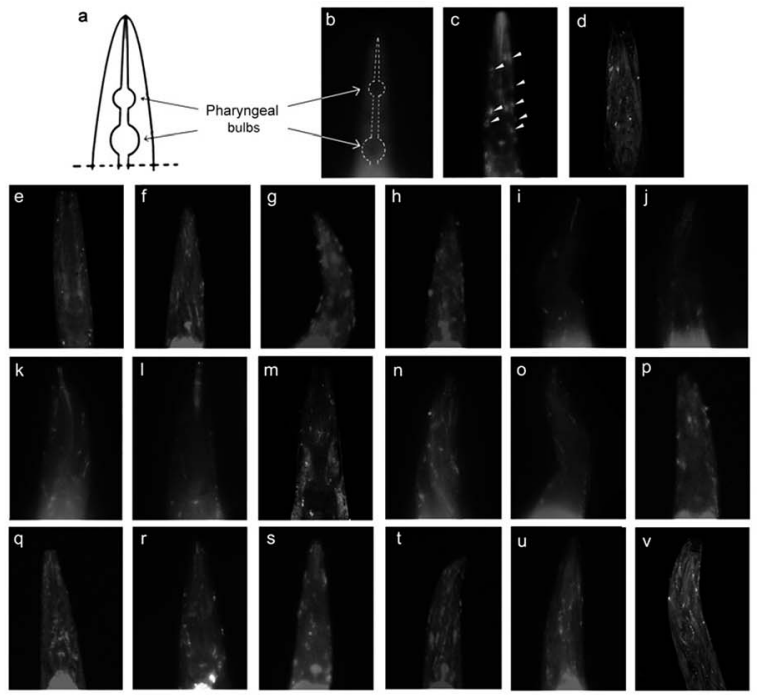

C

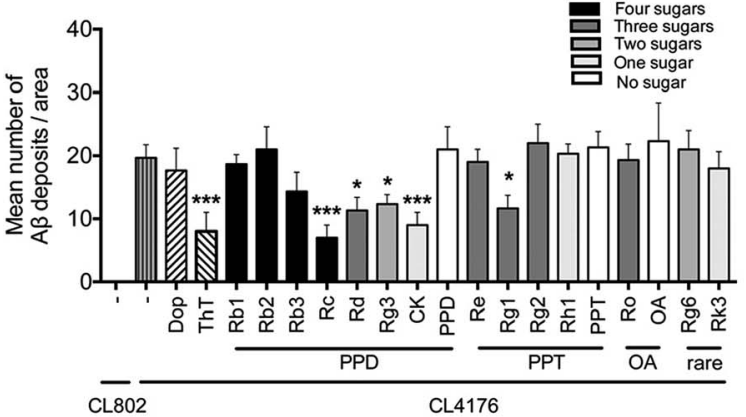

Fig. 1 Effects of ginsenosides on $A \beta$ deposits in transgenic C. elegans. (A) An illustrative diagram indicating the time duration of treatment and detectable $A \beta$ deposits stain. Synchronized CL2006 or CL2109 were placed at $16{ }^{\circ} \mathrm{C}$ for $72 \mathrm{~h}$, and then treated with vehicle (0.1\% DMSO), Dop $(50 \mu \mathrm{M})$, ThT $(50 \mu \mathrm{M})$ or ginsenosides $(50 \mu \mathrm{M})$ for $48 \mathrm{~h}$. The worms were then stained with Methoxy-X04 (1 mM in $10 \mathrm{mM}$ Tris, $\mathrm{pH}$ 8.0) for $4 \mathrm{~h}$ at room temperature. After staining, worms were subsequently returned to seeded NGM plates for $12 \mathrm{~h}$ to allow destaining via normal metabolism. Stained worms were mounted and photographed by using fluorescence microscope. (B) The schematic diagram indicates position of pharyngeal bulbs (a) and representative images of fluorescence in CL2109 (b), CL2006 treated with vehicle (0.1\% DMSO) (c), $\operatorname{Dop}(\mathrm{d}), \operatorname{ThT}(\mathrm{e})$ or ginsenoside Rb1 (f), Rb2 (g), Rb3 (h), Rc (i), Rd (j), Rg3 (k), $C K(l), \operatorname{PPD}(m), \operatorname{Re}(n), \operatorname{Rg} 1$ (o), Rg2 (p), Rg2 (q), Rh1 (r), PPT (s), Ro (s), $\mathrm{OA}(\mathrm{t}), \mathrm{Rg} 6(\mathrm{u})$ and $\mathrm{Rk} 3(\mathrm{v})$ at $50 \mu \mathrm{M}$ in $\mathrm{M} 9$ buffer. White arrows indicate $A \beta$ deposits in head region. (C) Quantitative analysis of $A \beta$ deposits in the transgenic $C$. elegans CL2006 treated with different ginsenosides for $48 \mathrm{~h}$. The quantity is expressed as mean number of $A \beta$ deposits/ anterior area of worm ( $n=25$ for each analysis). Error bars indicate SD. \#\#\# $p<0.001, * * * p<0.001$ compared with CL2006 vehicle treated group.

nematode growth medium (NGM) seeded with E. coli (OP50) as a food source. ${ }^{32}$ To prepare age-synchronized populations, nematodes reaching reproductive maturity at 3 days of age were transferred to fresh NGM plates and allowed to lay eggs for $4 \mathrm{~h}$. 
A

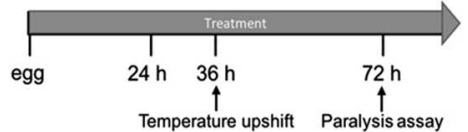

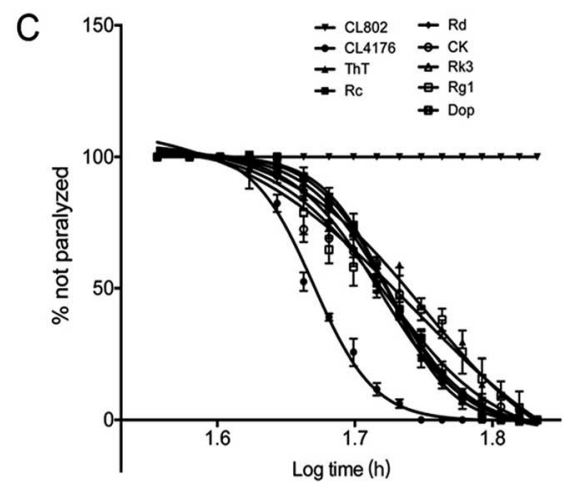

B

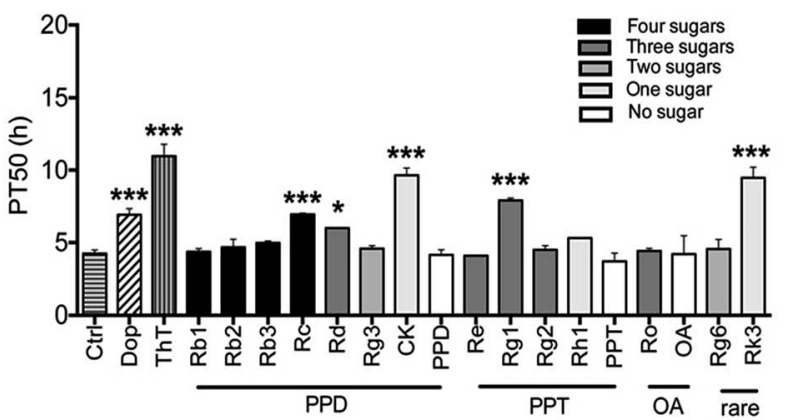

Fig. 2 Effects of ginsenosides on $A \beta$ induced paralysis in the transgenic $C$. elegans (A) an illustrative diagram indicating the time duration of treatment and paralysis assays in CL4176 worms treated with different compounds (50 $\mu$ M). Synchronized eggs of CL802 or CL4176 worms were maintained at $16{ }^{\circ} \mathrm{C}$ on the NGM plates. CL4176 worms were treated with vehicle (0.1\% DMSO), Dop $(50 \mu M)$, ThT (50 $\mu$ M) or ginsenosides (50 $\mu$ M) for $36 \mathrm{~h}$. Then the temperature was increased to $25^{\circ} \mathrm{C}$ to induce $A \beta$ expression. Paralysis was scored at $2 \mathrm{~h}$ intervals. (B) The paralysis assays were quantified for mean time duration at which $50 \%$ worms were paralyzed ( $\mathrm{PT}_{50}$ ) from the transgenic worms fed with or without the drugs. Values of $p$ were obtained from 6 independent assays for the worms compared with control group (treated with vehicle). Error bars indicate SD. ${ }^{\# \# \# ~} p<$ $0.001, * p<0.05, * * * p<0.001$ compared with Ctrl group. (C) Time course of paralysis assays in CL802 or CL4176 fed with different drugs. Paralysis in the transgenic strain CL4176 is attributable to $A \beta$ expression compared with the control strain CL802, which does not express $A \beta$ transgene. Data are expressed as percentage of nonparalyzed worms from at least three independent assays of 100 worms in each experiment.

The synchronized eggs were cultured on fresh NGM plates. All drug treatments were performed in $34.8 \times 10 \mathrm{~mm}$ Petri plates containing NGM agar. Placed $500 \mu \mathrm{L}$ of compound solution onto each plate and distributed over the surface of NGM agar. Allowed solution to dry overnight at room temperature and followed by adding $100 \mu \mathrm{L}$ of $E$. coli (OP50) onto the center of plate. The worms were fed with the drugs either from $72 \mathrm{~h}$ of age or starting from the egg.

\subsection{Fluorescence staining of $A \beta$ in C. elegans}

Methoxy-X04 (MedChem Express, USA) is a Congo-red derivative and has been widely used for in vivo imaging of $\mathrm{A} \beta$ plaques. ${ }^{33}$ In this study, methoxy-X04 was used for fluorescence staining of A $\beta$ deposit in C. elegans. Transgenic worms, CL2006 and its control strain CL2109, were synchronized and plated on NGM plate with OP50 at $16{ }^{\circ} \mathrm{C}$ for $72 \mathrm{~h}{ }^{34}$ Then worms were transferred into NGM plates, which contain vehicle (DMSO, 0.1\%) or compounds solution for $48 \mathrm{~h}$. Live worms were collected and incubated in methoxy-X04 solution $(1 \mathrm{mM}$ in $10 \mathrm{mM}$ Tris, $\mathrm{pH}$ 8.0) for four hours at room temperature. After staining, worms were subsequently transferred to seeded NGM plates for $12 \mathrm{~h}$ to allow destaining via normal metabolism. Stained worms were mounted and photographed by using fluorescence microscope
(Nikon, Japan). The quantitative analysis of $\mathrm{A} \beta$ deposits was performed as described before. ${ }^{35}$

\subsection{Paralysis assay}

Paralysis assay was performed as described before. ${ }^{36}$ Briefly, Petri plates $(34.8 \times 10 \mathrm{~mm})$ containing NGM agar were supplemented with vehicle ( $0.1 \%$ DMSO) or compounds solution. The strain CL4176 and CL802 maintained at $16{ }^{\circ} \mathrm{C}$ were eggsynchronized onto NGM plates containing vehicle or drug. Transgene expression was induced by upshifting the culture temperature from $16{ }^{\circ} \mathrm{C}$ to $25{ }^{\circ} \mathrm{C}$ at the $36 \mathrm{~h}$ after egg laying (third-stage larvae). Culture temperature was kept at $25^{\circ} \mathrm{C}$ until the end of the paralysis assay. Nematodes were scored as paralyzed if they failed to move during observation and exhibited 'halos' of cleared bacteria around their heads (indication of insufficient body movement to access food) ${ }^{37}$ Paralysis was scored at $2 \mathrm{~h}$ intervals.

\subsection{Odorant preference assay}

Chemotaxis assay was performed as described before ${ }^{31}$ with slightly modified. CL2241 and its control strain CL2122 were treated with or without Dop, VitE or ginsenosides starting from egg at $16{ }^{\circ} \mathrm{C}$ for $36 \mathrm{~h}$, and then the culture temperature was 
A

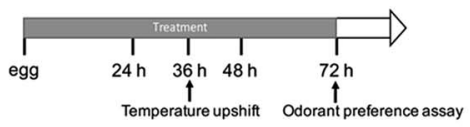

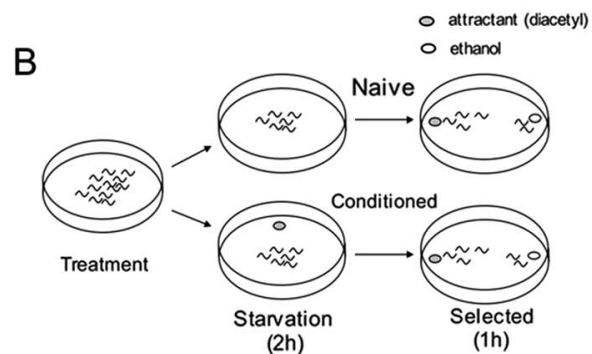

(2h)

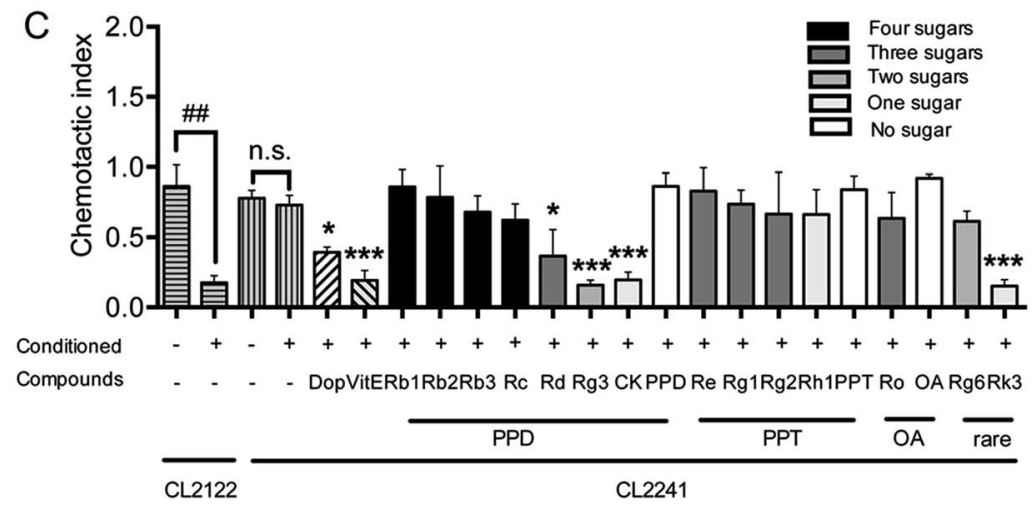

Fig. 3 Effects of ginsenosides on neuronal $A \beta$ expression induced defect in associative learning capacity (A) an illustrative diagram indicating the time duration of treatment and paralysis assays in CL2122 or CL2241 worms. (B) Schematic illustration of odorant preference associative learning assay. At the end of drug treatment (Dop, $50 \mu \mathrm{M}$; ThT, $50 \mu \mathrm{M}$; or ginsenosides, $50 \mu \mathrm{M}$ ), worms were starved for $2 \mathrm{~h}$ either in the presence (conditioned) or absence (naïve) of the odorant diacetyl and subsequently subjected to a chemotaxis assay. (C) The chemotaxis index in the control strain (CL2122) and the transgenic strain (CL2241) were scored as mentioned in method part. Each experiment was performed in triplicate. Error bars represent the standard error. ${ }^{\# \#} p<0.01 .{ }^{*} p<0.05,{ }^{* * *} p<0.001$ compared with relevant naïve group.

upshifted to $23^{\circ} \mathrm{C}$ to induce expression of $\mathrm{A} \beta$ and incubated for another $36 \mathrm{~h}$. Worms were then collected, washed with M9 buffer $^{38}$ three times, and transferred to NGM plates without food for $2 \mathrm{~h}$. To condition the worms, $2 \mu \mathrm{L}$ of odorant $(0.01 \%$ diacetyl in $95 \%$ ethanol) was placed in the lid of the plates and then the plates were inverted. Naïve worms were starved in the absence of diacetyl, synchronously. At the end of starvation, all worms were subjected to chemotaxis assay. ${ }^{39}$ Assays were performed in $100 \mathrm{~mm}$ plates containing $1.9 \%$ agar, $1 \mathrm{mM} \mathrm{CaCl}_{2}$, $1 \mathrm{mM} \mathrm{MgSO}_{4}$, and $25 \mathrm{mM}$ phosphate buffer, $\mathrm{pH}$ 6.0. For chemotaxis assay, $1 \mu \mathrm{L}$ of diacetyl was placed at $2.5 \mathrm{~cm}$ from the center of the plate on one side, and $1 \mu \mathrm{L}$ of $95 \%$ ethanol was added on the opposite. Twenty worms were quickly transferred to the center of the plate, then an additional $1 \mu \mathrm{L}$ of odorant or $95 \%$ ethanol along with $1 \mu \mathrm{L}$ of $1 \mathrm{M}$ sodium azide were added to the original spot. Assay plates were incubated at $20^{\circ} \mathrm{C}$ for $1 \mathrm{~h}$ and chemotaxis index (CI) was scored.

Chemotaxis index is defined as follows: (number of worms at the attractant spot - number of worms at the control spot)/total number of worm

\subsection{Oxidative free radicals assay}

Intracellular oxidative free radicals (ROS) in C. elegans and cells were measured using 2,7-dichlorofluorescein diacetate (DCFDA) (Beyotime, Shanghai, China) as described before. ${ }^{35}$ For in vitro assay, SH-SY5Ysw cells were pretreated with vehicle (DMSO, $0.1 \%$ ) or indicated compounds for $48 \mathrm{~h}$, then stimulated for transgene $A \beta$ expression by addition of $1 \mu \mathrm{M}$ butyric acid (BA) (Sigma, St. Louis, MO, USA) for $24 \mathrm{~h}$. For in vivo assay, age synchronized groups of CL4176 and CL802 were treated with compounds as mentioned before. After $36 \mathrm{~h}$ long temperature upshift, at least 60 worms from each group were collected into $100 \mu \mathrm{L}$ of PBS with 1\% Tween 20 (PBST). Worms were subjected to homogenization and sonication to break up the outer cuticle. Both cell and worm samples were incubated with DCF-DA solution $\left(50 \mu \mathrm{M}\right.$ in PBS) at $37{ }^{\circ} \mathrm{C}$ for $30 \mathrm{~min}$. Fluorescence was measured by microplate fluorescence reader with the excitation at $485 \mathrm{~nm}$ and emission at $530 \mathrm{~nm}$.

\subsection{Cell culture}

All cells were cultured at $37{ }^{\circ} \mathrm{C}$ in a humid incubator with $5 \%$ $\mathrm{CO}_{2}$. The human neuroblastoma cell line SH-SY5Y was purchased from American Type Culture Collection (Manassas, VA, USA) and grown in Dulbecco's modified Eagles Medium (DMEM) supplemented with $10 \%$ fetal bovine serum (FBS) and $1 \%$ penicillin/streptomycin (Gibco, New York, USA). The transgenic cell line SH-SY5Ysw cells that stably expressing amyloid $\beta$ protein precursor (APP) with the Swedish mutation were purchased from Velox Pharmaceutics, Inc. (VLXSTC0002, Changzhou, China) and grown in DMEM supplemented with 10\% FBS, 1\% penicillin/streptomycin (Gibco, New York, USA) and $200 \mu \mathrm{g} \mathrm{mL}{ }^{-1} \mathrm{G} 418$ (Invitrogen, California, USA). ${ }^{40}$

The laboratory animal facility in the animal center has been accredited by Association for Assessment and Accreditation of 
A

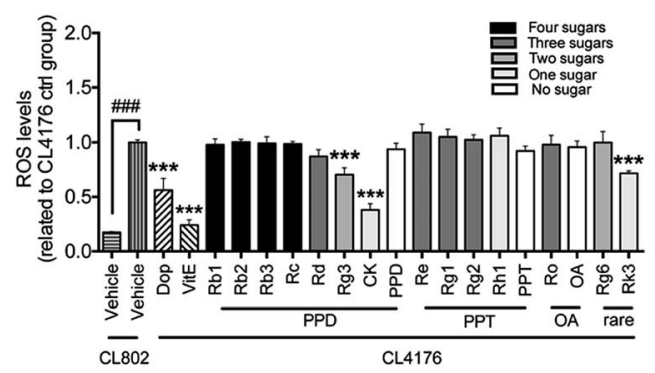

B

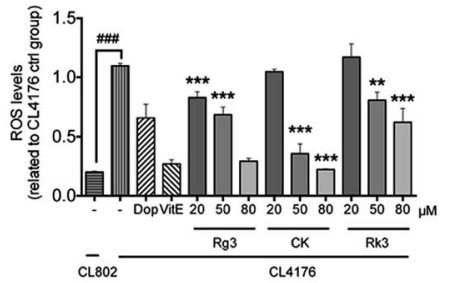

C

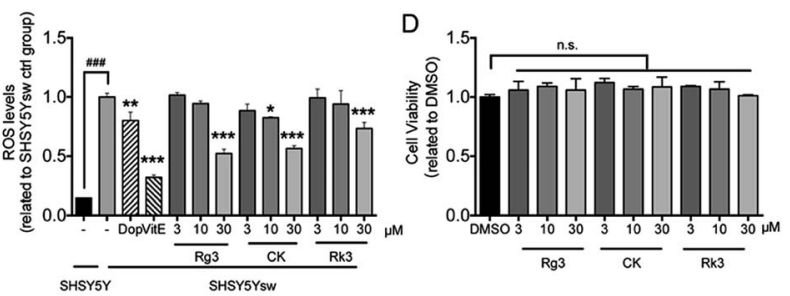

Fig. 4 Effect of ginsenosides on the production of ROS Levels of ROS in the $C$. elegans fed with different ginsenoside (A) or ginsenoside 20(S)-Rg3, CK and Rk3 at different concentrations (B). Age-synchronized groups of transgenic C. elegans strain CL802 or CL4176 at $16^{\circ} \mathrm{C}$ for $36 \mathrm{~h}$ were temperature upshifted to $25^{\circ} \mathrm{C}$ for $36 \mathrm{~h}$ followed by the DCF assay for ROS as described in Method section. At least 60 worms from each group were analyzed for the levels of ROS. Results are expressed as fold change of fluorescence in CL4176 control group (treated with vehicle), which is set as 1 . Error bars represent the stan-

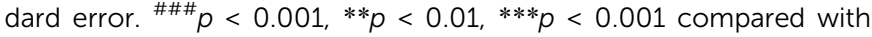
CL4176 control group. (C) Cells were pretreated with vehicle $(0.1 \%$ DMSO) or ginsenoside Rg3, CK, Rk3 at different concentrations for $48 \mathrm{~h}$ followed by administrated with BA $(1 \mu \mathrm{M})$ for $24 \mathrm{~h}$ to stimulate the express of transgene. ROS levels in cells were measured as described in Method section. Results were obtained from 6 independent samples for each compound tested. (D) Primary neurons (DIV 8) were treated with Rg3, CK or Rk3 at different concentration for $24 \mathrm{~h}$ followed by measurement of cell viability through MTT assay. Error bars represent

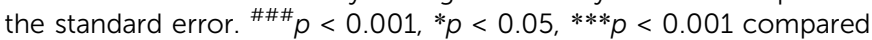
with $\mathrm{SH}-\mathrm{SY} 5 Y$ Sw vehicle treated group.

Laboratory Animal Care International. All experiments and animal care in this study were conducted in accordance with the Provision and General Recommendation of Chinese Experimental Animals Administration Legislation and approved by the Science and Technology Department of Jiangsu Province (SYXK (SU) 2016-0011). Primary neurons were isolated as described before. ${ }^{41}$ Briefly, Pregnant Sprague Dawley rat were sacrificed and E18 embryos were removed from abdomen. Cerebral cortices were separated and placed into ice cold Hank's balanced salt solution. After the scalp and skull were peeled away, cortex tissue was digested by $0.05 \%$ trypsin-EDTA (Gibco, New York, USA) for $10 \mathrm{~min}$ at $37^{\circ} \mathrm{C}$. The digestion was stopped by addition of Neurobasal medium containing $0.1 \mathrm{mg} \mathrm{mL}$
DNase I (Gibco, New York, USA). Digested tissues were centrifuged at $1500 \mathrm{rpm}$ for $5 \mathrm{~min}$ at $4{ }^{\circ} \mathrm{C}$ after gentle trituration and resuspended in a Neurobasal culture medium supplemented with 2\% B27 (Invitrogen, Grand Island, NY) and $0.5 \mathrm{mM}$ GlutaMax (Life Technologies, USA), and then filtered through a 70 $\mu \mathrm{m}$ nylon cell restrainer. Cells were diluted to $1 \times 10^{6}$ cells $\mathrm{mL}^{-1}$ and seeded into poly-L-lysine (Beyotime, Shanghai, China) coated plates.

\subsection{Cell viability assay}

Assessment of primary cortical neurons viability was performed by MTT [3-(4,5-dimethylthiazol-2-yl)-2,5-diphenyl-2 $H$-tetrazolium bromide] (Sigma, St. Louis, MO, USA) assay on day in vitro (DIV) 8. Stock solutions of ginsenosides in DMSO were diluted to final concentration and administrate to cells for $24 \mathrm{~h}$. Then, MTT ( $5 \mathrm{mg} \mathrm{ml}^{-1}$ in PBS) were added to culture medium in each wells to make the final concentration at $0.5 \mathrm{mg} \mathrm{ml}^{-1}$ and incubated for another $4 \mathrm{~h}$. The medium was removed and 200 $\mu \mathrm{L}$ of DMSO was added to dissolve the formazan crystals. Absorbance values were measured at $490 \mathrm{~nm}$.

\subsection{Statistical analysis}

Data were presented as mean \pm standard deviation (SD). Difference between two groups was analyzed for statistical significance by independent Student's $t$ test. Difference between three or more groups was using the one-way ANOVA followed by Dunnett's test. $p<0.05$ was considered significant.

\section{Results}

\subsection{Effects of ginsenosides on $\mathrm{A} \beta$ deposits in $\mathrm{AD}$ C. elegans}

The $\mathrm{A} \beta_{42}$ peptides are highly fibrillogenic and readily aggregate. They could assemble into different forms including monomer, oligomer, fibrillar and finally made the insoluble plaques. ${ }^{2}$ To validate whether ginsenosides affect $A \beta$ deposits formation in vivo, the transgenic C. elegans line CL2006 worms in which human $A \beta_{42}$ peptide is expressed in muscle cells and form deposits $^{42}$ were treated with Dop, ThT or ginsenosides. CL2109 was used as a genetic background control strain of CL2006. Worms were subjected to $\mathrm{A} \beta$ staining using methoxy-X04 at the end of drug treatment. The number of $A \beta$ deposits was scored in the worm's head region, which is separated from the rest of the body by the pharyngeal bulb (Fig. 1Ba and b). As shown in Fig. $2 A c, A \beta$ deposits (white arrowheads) were detected in the CL2006 worms but not in CL2109 (Fig. 1Bb) as observed before. ${ }^{43}$ Dop and ThT were served as positive control in this assay. Dop is an inhibitor of acetylcholinesterase and widely used in clinical treatment of mild to moderate AD. ThT could bind to $A \beta$ and ameliorate $A \beta$ induced toxicity in C. elegans through regulating the protein homeostasis network. ${ }^{37}$ The number of $A \beta$ deposits was significantly reduced in worms treated with ThT (Fig. 1Be), Rc (Fig. 1Bi), Rd (Fig. 1Bj), Rg3 (Fig. 1Bk), CK (Fig. 1Bl) and Rg1 (Fig. 1Bo). The average number of $A \beta$ deposits from 25 worms in each group is shown in Fig. 2C. 


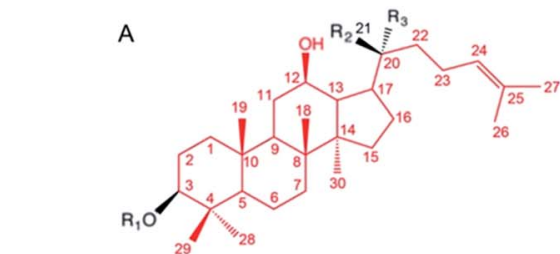

\begin{tabular}{ccccc}
\hline No. & Identification & $\mathrm{R}_{1}$ & $\mathrm{R}_{2}$ & $\mathrm{R}_{3}$ \\
\hline 1 & $\mathrm{Rb} 1$ & $-\mathrm{glc}(2-1) \mathrm{glc}$ & $-\mathrm{Oglc}(6-1) \mathrm{glc}$ & $-\mathrm{CH}_{3}$ \\
2 & $\mathrm{Rb} 2$ & $-\mathrm{glc}(2-1) \mathrm{glc}$ & $-\mathrm{Oglc}(6-1) \mathrm{arap}$ & $-\mathrm{CH}_{3}$ \\
3 & $\mathrm{Rb} 3$ & $-\mathrm{glc}(2-1) \mathrm{glc}$ & $-\mathrm{Oglc}(6-1) \times y l$ & $-\mathrm{CH}_{3}$ \\
4 & $\mathrm{Rc}$ & $-\mathrm{glc}(2-1) \mathrm{glc}$ & $-\mathrm{Oglc}(6-1) \operatorname{araf}$ & $-\mathrm{CH}_{3}$ \\
5 & $\mathrm{Rd}$ & $-\mathrm{glc}(2-1) \mathrm{glc}$ & $-\mathrm{Oglc}$ & $-\mathrm{CH}_{3}$ \\
6 & $20(\mathrm{~S})-\mathrm{Rg} 3$ & $-\mathrm{glc}(2-1) \mathrm{glc}$ & $-\mathrm{OH}$ & $-\mathrm{CH}_{3}$ \\
7 & $\mathrm{CK}$ & $-\mathrm{H}$ & $-\mathrm{Oglc}$ & $-\mathrm{CH}_{3}$ \\
8 & $\mathrm{PPD}$ & $-\mathrm{H}$ & $-\mathrm{OH}$ & $-\mathrm{CH}_{3}$ \\
\hline
\end{tabular}

C

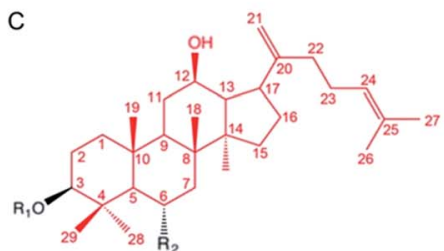

\begin{tabular}{cccc}
\hline No. & Identification & $\mathrm{R}_{1}$ & $\mathrm{R}_{2}$ \\
\hline 1 & $\mathrm{Rk} 3$ & $-\mathrm{H}$ & - Oglc \\
2 & $\mathrm{Rg} 6$ & $-\mathrm{H}$ & $-\mathrm{Oglc}(2-1)$ rha \\
\hline
\end{tabular}

B

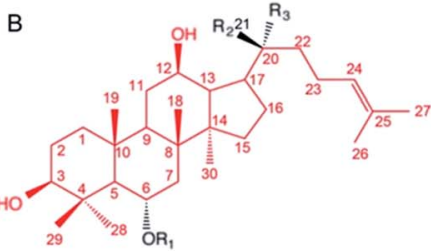

\begin{tabular}{ccccc}
\hline No. & Identification & $\mathrm{R}_{1}$ & $\mathrm{R}_{2}$ & $\mathrm{R}_{3}$ \\
\hline 1 & $\mathrm{Re}$ & $-\mathrm{glc}(2-1) \mathrm{rha}$ & $-\mathrm{Oglc}$ & $-\mathrm{CH}_{3}$ \\
2 & $\mathrm{Rg} 1$ & $-\mathrm{glc}$ & $-\mathrm{Oglc}$ & $-\mathrm{CH}_{3}$ \\
3 & $\mathrm{Rg} 2$ & $-\mathrm{glc}(2-1) \mathrm{rha}$ & $-\mathrm{OH}$ & $-\mathrm{CH}_{3}$ \\
4 & $20(\mathrm{~S})-\mathrm{Rh} 1$ & $-\mathrm{glc}$ & $-\mathrm{OH}$ & $-\mathrm{CH}_{3}$ \\
5 & $\mathrm{PPT}$ & $-\mathrm{H}$ & $-\mathrm{OH}$ & $-\mathrm{CH}_{3}$ \\
\hline
\end{tabular}

D

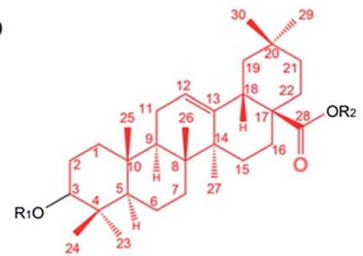

\begin{tabular}{cccc}
\hline No. & Identification & $\mathrm{R}_{1}$ & $\mathrm{R}_{2}$ \\
\hline 1 & ginsenoside Ro & $-\mathrm{glc}(1-2) \mathrm{glc}$ & $-\mathrm{glc}$ \\
2 & oleanolic acid & $-\mathrm{H}$ & $-\mathrm{H}$ \\
\hline
\end{tabular}

Fig. 5 Chemical structures of the studied ginsenosides. (A) Protopanaxadiol (PPD)-type ginsenosides, (B) protopanaxatriol (PPT)-type ginsenosides, (C) rare ginsenosides, (D) oleanane-type ginsenosides. glC, $\beta$-D-glucose; rha, $\alpha$-L-rhamnose; arap, $\alpha$-L-arabinose (pyranose); araf, $\alpha$-Larabinose (furanose); xyl, $\beta$-D-xylopyranose. The red line indicated the aglycone structure.

\subsection{Effects of ginsenosides on $A \beta$ induced paralysis in the transgenic C. elegans}

To determine whether ginsenosides specifically protects against $\mathrm{A} \beta$ induced toxicity in vivo, ginsenosides or aglycones were administrated to CL4176, the transgenic C. elegans line in which human $A \beta_{42}$ peptide expressed in muscle cells and induce an $A \beta$ dependent paralysis phenotype. ${ }^{44}$ The process of paralysis assay was described in Fig. 2A. Synchronized eggs of CL4176 and CL802 (used as genetic background control strain of CL4176) were placed on NGM plates containing vehicle, Dop $(50 \mu \mathrm{M})$, ThT $(50 \mu \mathrm{M})$ or different ginsenosides $(50 \mu \mathrm{M})$ for $36 \mathrm{~h}$, followed by upshifting temperature from $16{ }^{\circ} \mathrm{C}$ to $25{ }^{\circ} \mathrm{C}$ to induce $\mathrm{A} \beta_{42}$ expression. PT50 is defined as the time interval from the onset of paralysis to $50 \%$ of the worms were paralyzed ${ }^{45}$ and used for

Table 2 Possible structure-activity relationships of PPD and PPT type ginsenosides against A $\beta^{a}$

\begin{tabular}{|c|c|c|c|c|c|c|c|c|c|c|}
\hline \multirow[b]{2}{*}{ Identification } & \multirow[b]{2}{*}{ Aglycone } & \multirow[b]{2}{*}{ No. of sugars } & \multicolumn{4}{|c|}{ Position of sugars } & \multicolumn{4}{|c|}{ Anti-A $\beta$ activity } \\
\hline & & & C-3 & C-6 & $\mathrm{C}-12$ & $\mathrm{C}-20$ & Paralysis & Stain & $\mathrm{CI}$ & ROS \\
\hline $\mathrm{Rb} 2$ & & 4 & 2 & 0 & $1-\mathrm{OH}$ & 2 & n.s. & n.s. & n.s. & n.s. \\
\hline Rb3 & & 4 & 2 & 0 & $1-\mathrm{OH}$ & 2 & n.s. & n.s. & n.s. & n.s. \\
\hline $\mathrm{Rc}$ & & 4 & 2 & 0 & $1-\mathrm{OH}$ & 2 & $* * *$ & $* * *$ & n.s. & n.s. \\
\hline CK & & 1 & 1 & 0 & $1-\mathrm{OH}$ & 0 & $* * *$ & $* * *$ & $* * *$ & $* * *$ \\
\hline PPD & & 0 & 1-OH & 0 & $1-\mathrm{OH}$ & 0 & n.s. & n.s. & n.s. & n.s. \\
\hline $\mathrm{Re}$ & PPT & 3 & $1-\mathrm{OH}$ & 2 & $1-\mathrm{OH}$ & $1-\mathrm{OH}$ & n.s. & n.s. & n.s. & n.s. \\
\hline Rg1 & & 2 & 1-OH & 1 & $1-\mathrm{OH}$ & 1 & $* * *$ & $*$ & n.s. & n.s. \\
\hline $\mathrm{Rg} 2$ & & 3 & 1 & 2 & $1-\mathrm{OH}$ & 1-OH & n.s. & n.s. & n.s. & n.s. \\
\hline $20(S)-\mathrm{Rh} 1$ & & 1 & $1-\mathrm{OH}$ & 1 & $1-\mathrm{OH}$ & $1-\mathrm{OH}$ & n.s. & n.s. & n.s. & n.s. \\
\hline
\end{tabular}

$a * p<0.05,{ }^{* * *} p<0.001$ compared with model control group. 
Table 3 Possible structure-activity relationships of rare type ginsenosides against $A \beta^{a}$

\begin{tabular}{|c|c|c|c|c|c|c|c|c|c|}
\hline & No. of sugars & \multicolumn{4}{|c|}{ Position of sugars } & \multicolumn{4}{|c|}{ Anti-A $\beta$ activity } \\
\hline Rg6 & 2 & $1-\mathrm{OH}$ & 2 & $1-\mathrm{OH}$ & 0 & n.s. & n.s. & n.s. & n.s. \\
\hline
\end{tabular}

quantitative analysis. As shown in Fig. 2B and C, ThT treatment significantly delay $\mathrm{A} \beta$ induced paralysis which was consistent with the data reported previously, ${ }^{37}$ similarly, Dop. Rc, Rd, CK, Rg1 and Rk3 exhibited a significant delay of paralysis (Fig. 2B and $\mathrm{C}$ ).

\subsection{Effects of ginsenosides on neuronal $\mathbf{A} \beta$ expression induced defect in associative learning capacity}

Transgenic worm in which human $A \beta_{42}$ peptide is expressed in neuronal cells was described and used for researching the influences of $\mathrm{A} \beta$ on neurological functions. ${ }^{31}$ The chemotaxis response in C. elegans is related to activation of several sensory neurons and interneurons to stimulate the motor neurons. ${ }^{46}$ This innate behavior was paired with a conditioned stimulant and can be utilized to assess associative learning ability. ${ }^{31}$ Chemotaxis index (CI) value is defined as the fraction of worms that are able to arrive at the location of the attractants (diacetyl in this study), ${ }^{47}$ and is used to investigate the effects of ginsenosides on the performance of chemotaxis behavior in the present study. The transgenic strain CL2241 that has inducible expression of human $A \beta_{42}$ in the neurons ${ }^{30}$ was used for investigating the effects of ginsenosides on neuronal $A \beta$ expression induced defect in associative learning capacity. Synchronized eggs of CL2241 and CL2122 (used as genetic background control strain of CL2241) were placed on NGM plates containing vehicle $(0.1 \%$ DMSO) or different compounds for $36 \mathrm{~h}$, followed by upshifting the temperature from $16{ }^{\circ} \mathrm{C}$ to $23{ }^{\circ} \mathrm{C}$ to induce $\mathrm{A} \beta_{42}$ expression for another $36 \mathrm{~h}$ before odorant assay (Fig. 3A). Worms from the same treated plates were distributed equally into conditioned group or naïve group. Conditioned worms were starved for $2 \mathrm{~h}$ in the presence of diacetyl. Naïve worms were starved simultaneously in the absence of odorant. Then both groups were subjected to odorant preference assay for $1 \mathrm{~h}$ (Fig. 3B).

As shown in Fig. 3C, both CL2241 and CL2122 naïve worms preferred diacetyl though the transgenic worms displayed a slightly lower basal affinity for the diacetyl, indicating that the chemotaxis behavior of CL2241 worms was not impaired. After conditioned, CI of CL2122 worms was significantly decreased, while the CL2241 worms still prefer the attractants. These results indicated that CL2241 worms failed to associate the nutrient deprived environment with the environmental cue. CI of conditioned CL2241 worms were significantly decreased after Dop, VitE, Rg3, CK and Rk3 treatment, but not other ginsenosides or aglycones. These results indicated that Dop, VitE, Rg3, $\mathrm{CK}$ and Rk3 could ameliorate $\mathrm{A} \beta$ induced neurotoxicity.

\subsection{Effect of ginsenosides on the production of ROS}

Numerous evidences have shown that oxidative stress is associated with $\mathrm{A} \beta$ induced toxicity in $\mathrm{AD}^{\mathbf{4 8}}$. We first tested the effects of ginsenosides on the production of ROS in transgenic muscle expression A $\beta$ strain CL4176 and its control strain CL802. VitE is known to be an antoxidant and used in $\mathrm{AD}$ treatment. ${ }^{49}$ As shown in Fig. 4A, the untreated CL4176 worms exhibited increased levels of ROS compared with CL802 worms. Dop, VitE, Rg3, CK and Rk3 treatment before the induction of $\mathrm{A} \beta$ expression significantly prevent the elevation of ROS levels in CL4176 worms. We further found that Rg3, CK and Rk3 could attenuate the intracellular levels of ROS in CL4176 worms in a does-dependent manner (Fig. 4B).

Next, a transgenic mammal neuron cell line SH-SY5Ysw cells were used to further validate the effect of $20(S)$-Rg 3 , CK and Rk3 on internal expression of $A \beta$ induced ROS production. Dop and VitE were served as positive control. As shown in Fig. 4C, the levels of ROS were significantly higher in $A \beta$ secreting mutant cells SH-SY5Ysw compared to the wild type cells SH-SY5Y. Pretreatment with Rg3, CK and Rk3 for $48 \mathrm{~h}$ significantly attenuated the elevation of ROS levels in SH-SY5Ysw cells in a dose dependent manner. Moreover, none of these three saponins showed cytotoxicity in primary neurons (Fig. 4D).

\section{Discussion}

In the present study, we investigated the in vivo anti-A $\beta$ toxicity effects of different types of ginsenosides using transgenic $C$. elegans models. We found that (1) Rc, Rd, 20(S)-Rg3, CK that

Table 4 Possible structure-activity relationships of OA type ginsenosides against A $\beta$

\begin{tabular}{|c|c|c|c|c|c|c|c|c|c|}
\hline \multirow[b]{2}{*}{ Identification } & \multirow[b]{2}{*}{ Aglycone } & \multirow[b]{2}{*}{ No. of sugars } & \multicolumn{3}{|c|}{ Position of sugars } & \multicolumn{4}{|c|}{ Anti- $A \beta$ activity } \\
\hline & & & $\mathrm{C}-3$ & C-6 & C-28 & Paralysis & Stain & $\mathrm{CI}$ & ROS \\
\hline
\end{tabular}


belong to PPD type of ginsenoside, Rg1 that belongs to PPT type, could significantly reduce the formation of A $\beta$ deposits; (2) Rc, $\mathrm{Rd}, \mathrm{CK}, \mathrm{Rk} 3$ and Rg 1 inhibit $\mathrm{A} \beta$ induced paralysis, (3) 20(S)-Rg3, $\mathrm{CK}$ and $\mathrm{Rk} 3$ prevented $\mathrm{A} \beta$ induced chemotaxis associated learning dysfunction and reduce $A \beta$ induced ROS production both in whole organism and in transgenic mammal cells.

Among various in vitro and in vivo models of $\mathrm{AD}$, C. elegans is an ideal model organism for anti-AD drug discovery because of its well-developed genetics, short life cycle, simple structure of nervous system, and low dosage of drug consumption. Moreover, the absence of endogenous $\mathrm{A} \beta$ production in C. elegans make researchers could find direct role of $A \beta$ involvement in pathological behaviors. ${ }^{50}$ Since $\mathrm{AD}$ has been associated with aggregation of $A \beta$ and cell death in brain, different $A \beta$ transgenic $C$. elegans models were used in the present study to validate the potential anti-AD ability of ginsenosides.

In vitro studies have shown that $\mathrm{A} \beta$ aggregation start from monomers, then form the oligomer, protofibril and fibril. ${ }^{51}$ Recent studies showed that the oligomer, rather than the fibril or plaque, play as the main toxic species in the pathogenesis of AD. ${ }^{52,53}$ We found Rc, Rd, CK and Rg1 reduced $A \beta$ deposits in CL2006 worms through A $\beta$ staining assay (Fig. 1), and significantly delay the $A \beta$ induced paralysis (Fig. 2) in CL4176 worms. Combining the previous observation that paralysis occurs before detectable $\mathrm{A} \beta$ deposition formation in C. elegans, ${ }^{54}$ the paralysis delay effects of $\mathrm{Rc}, \mathrm{Rd}, \mathrm{CK}$ and $\mathrm{Rg} 1$ may related to the regulation of $A \beta$ oligomerization processes in worms. On the other hand, it has been found that inducible expression of human $\mathrm{A} \beta_{42}$ in muscle leads to an accumulation of autophagic vesicles in $C$. elegans which contribute to reducing $A \beta$ aggregation. ${ }^{55}$ Compounds that promote autophagy-lysosomal process could reduce $A \beta$ deposition and prevented $A \beta$ induced paralysis. ${ }^{56-58}$ It has been showed that $\mathrm{CK},{ }^{59} \mathrm{Rg} 3$ (ref. 60) could induce autophagy, which may explain the $A \beta$ deposits reducing effect of CK and Rg3 in CL4176 worms (Fig. 1B).

Considering the muscle specific $\mathrm{A} \beta$ expressing $C$. elegans model could not be specific reflect of the pathology in the AD brain. Therefore, we further used the transgenic C. elegans model that has inducible, pan-neuronal expression of $A \beta$ to evaluate in vivo effects of ginsenosides on neuronal $\mathrm{A} \beta$ toxicity. The relationship between $A \beta$ expression in neuron cells and defects in chemotaxis associated learning ability in transgenic C. elegans model has been well established. ${ }^{31}$ Dop, VitE, and four ginsenosides, Rd, Rg3, CK and Rk3 were found to significantly improve $\mathrm{A} \beta$ induced defect in associative learning capacity. Numerous studies suggested that the oxidative stress plays a key role in $A \beta$ induced neurotoxic and enhance the progression of $\mathrm{AD} .^{61,62}$ The oxidative stress is found increased in neurons that are exposed to $\mathrm{A} \beta$ oligomer $^{63}$ or neurons surrounded the $\mathrm{A} \beta$ plaque in the $\mathrm{AD}$ brain. ${ }^{64}$ Similarly, elevated ROS production was found increased in transgenic C. elegans expressing constitutive ${ }^{35}$ and inducible $A \beta .^{54}$ There is a positive correlation between ROS levels and muscle degeneration presenting as paralysis. ${ }^{54}$ Consistent with the previous report, ${ }^{45}$ we observed significantly increased ROS production in CL4176 worms in the present study. Recent studies have showed that the inhibitors of acetylcholinesterase altered redox homeostasis and attenuate $A \beta$ induced toxicity. ${ }^{65-67}$ Our results showed that both Dop and VitE, a known antioxidant, reduced ROS production in CL4176 and SHSY5Ysw transgenic cells (Fig. 4).

Among the ginsenosides that have been studied in the present research, Rg3, CK and Rk3 significantly decreased the in vivo ROS production in a dose dependent manner. The antioxidative effects of $\mathrm{Rg} 3, \mathrm{CK}$ and Rk3 were further confirmed in transgenic mammal neuronal cell line SH-SY5Ysw (Fig. 4). It has been reported that both Rg3 (ref. 68) and $\mathrm{CK}^{69}$ could suppress ROS production in bone marrow-derived macrophages and keratinocytes. It was also reported that CK induced autophagy and apoptosis in human colon cancer cells, but not in normal colon cells, through ROS generation. ${ }^{59}$ These results suggested that the effects of CK on ROS levels are cell-type specific. This might partially explain the effects of CK in SHSY5Ysw transgenic cells, since SHSY5Y is also a bone-marrow derived cell line. ${ }^{70}$ We found that CK also exhibited cytotoxicity in SHSY5Ysw cells at higher concentrations (Fig. S1†), which indicated that $\mathrm{CK}$ inhibits $\mathrm{A} \beta$ induced ROS production in a certain concentration range. It is interesting that $\mathrm{Rd}$ could delay $A \beta$ induced paralysis, reduce plaque formation and enhance chemotaxis associated learning ability in transgenic $C$. elegans models, but did not affect ROS production. The p38 MAPK pathway is involved in endoplasmic reticulum homeostasis and immune activation in $C$. elegans during stress. It has been reported that $\mathrm{Rd}$ could promote non-amyloidogenic pathway of amyloid precursor protein processing by regulating MAPK/PI3K pathway. ${ }^{23}$ Further research is needed to investigate whether $\mathrm{Rd}$ ameliorates $\mathrm{A} \beta$ induced toxicity through MAPK pathway in C. elegans.

Several in vitro studies suggest that the activity of ginsenosides have relationship with its structure. ${ }^{71-73}$ Takemoto et al. found that several PPD type saponins, but not PPT or oleanane type saponins, potentiated nerve growth factor (NGF) induced neurite outgrowth. ${ }^{74}$ Tohda et al. indicated that the PPD type saponins, including $\mathrm{Rb} 1$ and $\mathrm{Rb} 3$, enhanced axonal and dendritic formation in SK-N-SH cells, while the PPT, ocotillol and oleanane type saponins have no effect on the neurite outgrowth. ${ }^{75}$ In terms of ginsenosides studied in the present research (Fig. 5), four PPD type saponin, including Rc, Rd, Rg3 and CK, while only one PPT type saponin Rg1, that could significantly reduce $A \beta$ deposits (Fig. 2); three PPD type saponin that Rc, Rd, and CK, while only one PPT type saponin Rg1, that could significantly delay A $\beta$ induced paralysis (Fig. 3). Moreover, only PPD type saponin $\mathrm{Rg} 3$ and $\mathrm{CK}$, and rare type Rk3 ameliorated $\mathrm{A} \beta$ induced associative learning capacity deficiency and ROS production (Fig. 4 and 5). Our results suggested that different types of aglycone might contribute different anti-A $\beta$ activity. Sugar moiety is another key factor, which influences the activity of ginsenosides. ${ }^{71,76,77}$ The anti-A $\beta$ effects of tetrasaccharide glycoside, trisaccharide glycoside, disaccharide glycoside, monosaccharide glycoside and sapogenin in different C. elegans models were compared. Firstly, ginsenosides with less sugar moieties showed stronger efficacy in ameliorating $\mathrm{A} \beta$ induced toxicity in vivo. This might related to better absorption and cell membrane permeability of the ginsenosides with less sugar moieties and increased hydrophobicity. ${ }^{76}$ Secondly, the 
position of sugar linkage seems affect the anti-A $\beta$ activity of ginsenosides. As shown in Tables $2-4$, CK has one glucose moiety linked at its C-20 position and showed stronger anti-A $\beta$ activity than PPD. Rc, Rd, Rb1, Rb2 which have the same aglycone type and the same sugar moiety at C-3 position, but different types of sugar moiety linked at C-20 position. Both Rc and $\mathrm{Rd}$ showed anti-A $\beta$ activity in different models, while Rb1 and $\mathrm{Rb} 2$ have no significant effects. Rg1 and Re along with rare gensinosides Rk3 and Rg6 have different sugar moiety at C-6 position and showed different effects in C. elegans model. Moreover, PPD, PPT and OA showed insignificant effects in the present study. These results indicated that the moiety number and position of ginsenosides are crucial for their anti-A $\beta$ activity.

\section{Conclusions}

Our studies in C. elegans provided a systematic evaluation of ginsenosides on the anti-A $\beta$ effect in vivo. Moreover, based on the uncovered structure-activity relationships, we can select and modify specific ginsenosides to obtain new prodrug with superior anti-AD activity. However, given the differences in mammals and nematode physiology, more study is needed to determine the anti-AD activities of Rc, Rd, Rg3, CK, Rk3 and Rg1 in mammals. Of particular relevance are the questions of whether anti-A $\beta$ species of ginsenosides actually cross the blood brain barrier and whether these ginsenosides activation in the periphery can lead to physiological changes that impact the brain. Nevertheless, our studies add to the growing body of literature indicating that anti-oxidative is neuroprotective.

\section{Acknowledgements}

This work was supported by National Natural Science Foundation of China (No. 81573637, 81421005), "Twelfth-Five Years" Supporting Programs from the Ministry of Science and Technology of China (No. 2015ZX09101043). The authors would like to thank Prof. Christopher D. Link (Institute for Behavioral Genetics, University of Colorado) for generously providing $C$. elegans strains CL2109 and CL2241. The authors acknowledge Mr David Anderson (Southeast University) for his generous support with language editing of the manuscript, and Dr Bin $\mathrm{Wu}$ for his technical support with primary neuron culture.

\section{References}

1 K. Blennow, M. J. de Leon and H. Zetterberg, Lancet, 2006, 368, 387-403.

2 J. Hardy and D. J. Selkoe, Science, 2002, 297, 353-356.

3 D. M. Mann, T. Iwatsubo, N. J. Cairns, P. L. Lantos, D. Nochlin, S. M. Sumi, T. D. Bird, P. Poorkaj, J. Hardy, M. Hutton, G. Prihar, R. Crook, M. N. Rossor and M. Haltia, Ann. Neurol., 1996, 40, 149-156.

4 E. M. Snyder, Y. Nong, C. G. Almeida, S. Paul, T. Moran, E. Y. Choi, A. C. Nairn, M. W. Salter, P. J. Lombroso, G. K. Gouras and P. Greengard, Nat. Neurosci., 2005, 8, 1051-1058.
5 L. Jia and Y. Zhao, Curr. Med. Chem., 2009, 16, 2475-2484.

6 J. Y. Wan, Y. Fan, Q. T. Yu, Y. Z. Ge, C. P. Yan, R. N. Alolga, P. Li, Z. H. Ma and L. W. Qi, J. Pharm. Biomed. Anal., 2015, 107, 89-97.

7 H. J. Kim, P. Kim and C. Y. Shin, J. Ginseng Res., 2013, 37, 829.

8 K. Radad, G. Gille, L. Liu and W. D. Rausch, J. Pharmacol. Sci., 2006, 100, 175-186.

9 E. Tachikawa, K. Kudo, K. Harada, T. Kashimoto, Y. Miyate, A. Kakizaki and E. Takahashi, Eur. J. Pharmacol., 1999, 369, 23-32.

10 A. S. Attele, J. A. Wu and C. S. Yuan, Biochem. Pharmacol., 1999, 58, 1685-1693.

11 L. W. Qi, C. Z. Wang and C. S. Yuan, Nat. Prod. Rep., 2011, 28, 467-495.

12 M. H. Kim, Y. C. Lee, S. Y. Choi, C. W. Cho, J. Rho and K. W. Lee, J. Ginseng Res., 2011, 35, 497-503.

13 B.-H. Kim, S.-Y. Lee, H.-J. Cho, S.-N. You, Y.-J. Kim, Y.-M. Park, J.-K. Lee, M.-Y. Baik, C.-S. Park and S.-C. Ahn, Biol. Pharm. Bull., 2006, 29, 2472-2478.

14 J.-R. Choi, S.-W. Hong, Y. Kim, S.-E. Jang, N.-J. Kim, M. J. Han and D.-H. Kim, J. Ginseng Res., 2011, 35, 301-307.

15 T. F. Lee, Y. J. Shiao, C. F. Chen and L. C. Wang, Planta Med., 2001, 67, 634-637.

16 C. Tohda, N. Matsumoto, K. Zou, M. R. Meselhy and K. Komatsu, Neuropsychopharmacology, 2004, 29, 860-868.

17 N. Nishiyama, S. I. Cho, I. Kitagawa and H. Saito, Biol. Pharm. Bull., 1994, 17, 509-513.

18 B. Jiang, Z. Xiong, J. Yang, W. Wang, Y. Wang, Z. L. Hu, F. Wang and J. G. Chen, Br. J. Pharmacol., 2012, 166, 18721887.

19 N. Li, Y. Liu, W. Li, L. Zhou, Q. Li, X. Wang and P. He, J. Ginseng Res., 2016, 40, 9-17.

20 B. Lee, B. Sur, J. Park, S. H. Kim, S. Kwon, M. Yeom, I. Shim, H. Lee and D. H. Hahm, Biomol. Ther., 2013, 21, 381-390.

21 J. Liu, X. Yan, L. Li, Y. Zhu, K. Qin, L. Zhou, D. Sun, X. Zhang, R. Ye and G. Zhao, Neurochem. Res., 2012, 37, 2738-2747.

22 X. Zhang, M. Shi, R. Ye, W. Wang, X. Liu, G. Zhang, J. Han, Y. Zhang, B. Wang, J. Zhao, J. Hui, L. Xiong and G. Zhao, Neurochem. Res., 2014, 39, 1363-1373.

23 X. Yan, G. Hu, W. Yan, T. Chen, F. Yang, X. Zhang, G. Zhao and J. Liu, Life Sci., 2017, 168, 16-23.

24 Q. Wan, X. Ma, Z. J. Zhang, T. Sun, F. Xia, G. Zhao and Y. M. Wu, Mol. Neurobiol., 2017, 54, 2889-2900.

25 C. F. Wu, X. L. Bi, J. Y. Yang, J. Y. Zhan, Y. X. Dong, J. H. Wang, J. M. Wang, R. Zhang and X. Li, Int. Immunopharmacol., 2007, 7, 313-320.

26 Y. Z. Wang, J. Chen, S. F. Chu, Y. S. Wang, X. Y. Wang, N. H. Chen and J. T. Zhang, J. Pharmacol. Sci., 2009, 109, 504-510.

27 J. Qiu, W. Li, S. H. Feng, M. Wang and Z. Y. He, GMR, Genet. Mol. Res., 2014, 13, 3586-3598.

28 J. G. Hou, J. J. Xue, M. R. Lee, M. Q. Sun, X. H. Zhao, Y. N. Zheng and C. K. Sung, Biochem. Biophys. Res. Commun., 2013, 436, 104-109.

29 A. L. Lublin and C. D. Link, Drug Discovery Today: Technol., 2013, 10, e115-119. 
30 C. D. Link, Exp. Gerontol., 2006, 41, 1007-1013.

31 L. E. Dosanjh, M. K. Brown, G. Rao, C. D. Link and Y. Luo, J. Alzheimer's Dis., 2010, 19, 681-690.

32 H. F. Epstein and D. C. Shakes, Caenorhibditus Elegans: Modern Biological Analysis of an Organism, Academic Press, 1995.

33 J. Dong, R. Revilla-Sanchez, S. Moss and P. G. Haydon, Neuropharmacology, 2010, 59, 268-275.

34 L. Diomede, G. Cassata, F. Fiordaliso, M. Salio, D. Ami, A. Natalello, S. M. Doglia, A. De Luigi and M. Salmona, Neurobiol. Dis., 2010, 40, 424-431.

35 J. V. Smith and Y. Luo, J. Alzheimer's Dis., 2003, 5, 287-300. 36 V. Dostal and C. D. Link, J. Visualized Exp., 2010, 44, 2252.

37 S. Alavez, M. C. Vantipalli, D. J. Zucker, I. M. Klang and G. J. Lithgow, Nature, 2011, 472, 226-229.

38 W. B. Wood, Developmental Biology, New York, N.Y, 1985, 1988, vol. 5, pp. 57-78.

39 W. M. Nuttley, S. Harbinder and D. van der Kooy, Learning \& memory, Cold Spring Harbor, N.Y., 2001, vol. 8, pp. 170-181.

40 J. Wang, Z. Q. Shi, M. Zhang, G. Z. Xin, T. Pang, P. Zhou, J. Chen, L. W. Qi, H. Yang, X. Xu and P. Li, J. Alzheimer's Dis., 2015, 43, 465-477.

41 T. Pang, Y. J. Wang, Y. X. Gao, Y. Xu, Q. Li, Y. B. Zhou, L. Xu, Z. J. Huang, H. Liao, L. Y. Zhang, J. R. Gao, Q. Ye and J. Li, Acta Pharmacol. Sin., 2016, 37, 741-752.

42 C. D. Link, Proc. Natl. Acad. Sci. U. S. A., 1995, 92, 9368-9372.

43 C. D. Link, C. J. Johnson, V. Fonte, M. Paupard, D. H. Hall, S. Styren, C. A. Mathis and W. E. Klunk, Neurobiol. Aging, 2001, 22, 217-226.

44 C. D. Link, A. Taft, V. Kapulkin, K. Duke, S. Kim, Q. Fei, D. E. Wood and B. G. Sahagan, Neurobiol. Aging, 2003, 24, 397-413.

45 Y. Wu, Z. Wu, P. Butko, Y. Christen, M. P. Lambert, W. L. Klein, C. D. Link and Y. Luo, J. Neurosci., 2006, 26, 13102-13113.

46 O. Hobert, J. Neurobiol., 2003, 54, 203-223.

47 T. Matsuura, T. Sato and R. Shingai, Zool. Sci., 2005, 22, 1095-1103.

48 R. Sultana, M. Perluigi and D. A. Butterfield, Acta Neuropathol., 2009, 118, 131-150.

49 M. W. Dysken, P. D. Guarino, J. E. Vertrees, S. Asthana, M. Sano, M. Llorente, M. Pallaki, S. Love, G. D. Schellenberg, J. R. McCarten, J. Malphurs, S. Prieto, P. Chen, D. J. Loreck, S. Carney, G. Trapp, R. S. Bakshi, J. E. Mintzer, J. L. Heidebrink, A. Vidal-Cardona, L. M. Arroyo, A. R. Cruz, N. W. Kowall, M. P. Chopra, S. Craft, S. Thielke, C. L. Turvey, C. Woodman, K. A. Monnell, K. Gordon, J. Tomaska and G. Vatassery, Alzheimer's Dementia, 2014, 10, 36-44.

50 Y. Wu and Y. Luo, Curr. Alzheimer Res., 2005, 2, 37-45.

51 G. Bitan, M. D. Kirkitadze, A. Lomakin, S. S. Vollers, G. B. Benedek and D. B. Teplow, Proc. Natl. Acad. Sci. U. S. A., 2003, 100, 330-335.

52 A. Salminen, J. Ojala, T. Suuronen, K. Kaarniranta and A. Kauppinen, J. Cell. Mol. Med., 2008, 12, 2255-2262.
53 M. E. Larson and S. E. Lesne, J. Neurochem., 2012, 120(suppl. 1), 125-139.

54 J. Drake, C. D. Link and D. A. Butterfield, Neurobiol. Aging, 2003, 24, 415-420.

55 M. L. Florez-McClure, L. A. Hohsfield, G. Fonte, M. T. Bealor and C. D. Link, Autophagy, 2007, 3, 569-580.

56 C. Regitz, L. M. Dussling and U. Wenzel, Mol. Nutr. Food Res., 2014, 58, 1931-1940.

57 J. Li, X. Cui, X. Ma and Z. Wang, Exp. Gerontol., 2017, 89, 7886.

58 C. Regitz, E. Fitzenberger, F. L. Mahn, L. M. Dussling and U. Wenzel, Eur. J. Nutr., 2016, 55, 741-747.

59 A. D. Kim, K. A. Kang, H. S. Kim, D. H. Kim, Y. H. Choi, S. J. Lee, H. S. Kim and J. W. Hyun, Cell Death Dis., 2013, 4, e750.

60 M. Sun, C. Huang, C. Wang, J. Zheng, P. Zhang, Y. Xu, H. Chen and W. Shen, Biochem. Biophys. Res. Commun., 2013, 441, 169-174.

61 M. Pohanka, Curr. Med. Chem., 2014, 21, 356-364.

62 G. Perry, R. J. Castellani, K. Hirai and M. A. Smith, J. Alzheimer's Dis., 1998, 1, 45-55.

63 N. Izuo, T. Kume, M. Sato, K. Murakami, K. Irie, Y. Izumi and A. Akaike, ACS Chem. Neurosci., 2012, 3, 674-681.

64 H. Xie, S. Hou, J. Jiang, M. Sekutowicz, J. Kelly and B. J. Bacskai, Proc. Natl. Acad. Sci. U. S. A., 2013, 110, 79047909.

65 P. Atukeren, M. Cengiz, H. Yavuzer, R. Gelisgen, E. Altunoglu, S. Oner, F. Erdenen, D. Yuceakin, H. Derici, U. Cakatay and H. Uzun, Biomed. Pharmacother., 2017, 90, 786-795.

66 M. Pohanka, M. Hrabinova, F. Zemek, L. Drtinova, H. Bandouchova and J. Pikula, Neuroendocrinol. Lett., 2011, 32(suppl. 1), 95-100.

67 T. Zhang, F. Tian, J. Wang, S. Zhou, X. Dong, K. Guo, J. Jing, Y. Zhou and Y. Chen, Cell Stress Chaperones, 2015, 20, 787792.

68 Y. M. Shin, H. J. Jung, W. Y. Choi and C. J. Lim, Mol. Biol. Rep., 2013, 40, 269-279.

69 T. T. Cuong, C. S. Yang, J. M. Yuk, H. M. Lee, S. R. Ko, B. G. Cho and E. K. Jo, Life Sci., 2009, 85, 625-633.

70 R. A. Ross, B. A. Spengler and J. L. Biedler, J. Natl. Cancer Inst., 1983, 71, 741-747.

71 K. Quan, Q. Liu, J. Y. Wan, Y. J. Zhao, R. Z. Guo, R. N. Alolga, P. Li and L. W. Qi, Sci. Rep., 2015, 5, 8598.

72 G. X. Li and Z. Q. Liu, Food Chem. Toxicol., 2008, 46, 886-892. 73 S. Chae, K. A. Kang, U. Youn, J. S. Park and J. W. Hyun, J. Food Biochem., 2010, 34, 31-43.

74 Y. Takemoto, T. Ueyama, H. Saito, S. Horio, S. Sanada, J. Shoji, S. Yahara, O. Tanaka and S. Shibata, Chem. Pharm. Bull., 1984, 32, 3128-3133.

75 C. Tohda, N. Matsumoto, K. Zou, M. R. Meselhy and K. Komatsu, Jpn. J. Pharmacol., 2002, 90, 254-262.

76 S. A. Nag, J. J. Qin, W. Wang, M. H. Wang, H. Wang and R. Zhang, Front. Pharmacol., 2012, 3, 25.

77 H. Teng, L. Chen, T. Fang, B. Yuan and Q. Lin, J. Funct. Foods, 2017, 28, 306-313. 